\section{Summary}

Liver grafts are less sensitive to rejection than kidney, heart or pancreatic grafts. Tolerance of liver grafts has several aspects. Experimentally, in pigs and particularly in rats, liver allografts may become spontaneously tolerated and in rats may induce a donor specific state of transplantation tolerance. Liver grafts appear particularly resistant to lymphocytotoxic allogenic antibodies and also to xenogenic natural antibodies. In man, tolerance to liver allograft is less spectacular than in rat; however it is illustrated by the observation that liver allografts can be done without damage in case of a positive cross match or in case of an $\mathrm{ABO}$ incompatibility between donor and recipient.

\title{
Constitution d'un fichier de volontaires au don de moelle osseuse
}

Les greffes réalisées en France ont jusqu'à présent été faites à partir d'un donneur familial. En 1987 environ 500 greffes de moelle osseuse auront été effectuées. Des progrès récents permettent d'envisager la transfusion de cellules médullaires prélevées chez des donneurs non apparentés.

En effet, seuls $30 \%$ des patients devant bénéficier d'une greffe, auront dans leur fratrie un donneur HLA identique. Dans certaines indications et en particulier les leucémies myéloïdes chroniques, les aplasies, les déficits combinés sévères, des greffes à partir de donneur non apparenté ont été réalisées [1, 2]. Les premiers résultats étant encourageants [3], il est apparu nécessaire de constituer en France un fichier de volontaires au don de moelle osseuse comme il en existe dans d'autres pays notamment aux États-Unis [4-6].

Depuis environ deux ans, différents moyens ont été utilisés pour informer l'ensemble de la population française et non pas une certaine catégorie de donneurs potentiels qu'auraient pu être les donneurs de sang. Ces moyens ont transités par l'intermédiaire de bénévoles de la FFDOT (fédération française des donneurs d'organes et de tissus humains) et des pharmaciens ayant accepté de distribuer des formulaires d'information.

D'autres structures d'information se sont progressivement ajoutées telles que services d'accueil des centres de transfusion sanguine et associations de malades.
Chaque information est faite à deux niveaux : tout d'abord l'éventuel volontaire demande des renseignements qui lui permettront de connaître les réponses à la plupart des questions qu'il peut se poser: qu'est-ce que la moelle osseuse, quelles sont les contraintes d'un don anonyme et bénévole, etc., ensuite à sa demande il reçoit un formulaire comportant un consentement sur lequel le donneur s'engage à devenir volontaire jusqu'à l'âge de 50 ans pour un malade qu'il ne connaîtra jamais; ce consentement comporte par ailleurs des informations concernant le risque anesthésique et les délais de convocation dans le laboratoire devant réaliser les examens d'histocompatibilité. Seul le typage HLA$A$ et $B$ est fait pour entrer dans le fichier.

A ce jour, plus de 30000 demandes de renseignements ont été adressées par l'intermédiaire de la FFDOT. Les associations de malades, très actives, ont permis par ailleurs dans certaines régions le recueil d'un grand nombre de consentements. Un écueil pourtant est apparu très vite, c'est l'inadéquation entre le désir des familles de malades de constituer très rapidement ce fichier et la "nécessaire» lenteur pour la réalisation des groupes HLA. La seconde difficulté concerne les volontaires recrutés par des médias ne décrivant pas réellement les contraintes d'un tel don, c'est-à-dire don pour un malade anonyme: beaucoup veulent donner à un malade en 
particulier ou être volontaire à un moment limité dans le temps. Concernant les statistiques de recueil de consentements, il semble que le chiffre de 40000 soit atteint; mais beaucoup de volontaires ont signé leur consentement plusieurs mois auparavant et dans l'expérience d'un centre de la région parisienne plus d'un volontaire sur deux ne répond pas à la convocation pour typage HLA classe I.

Tous les volontaires au don de moelle osseuse sont convoqués par le laboratoire d'histocompatibilité du centre de transfusion proche de leur domicile pour un examen clinique et un interrogatoire pour déceler toute contre-indication au don qui sont en fait les mêmes que les contre-indications au don de sang.

Si le volontaire maintient sa décision, le groupe HLA et le groupe sanguin sont réalisés.

Les données comportant le nom, le prénom, la date de naissance, le numéro d'identification, les groupes $H L A-A$ et $B$, le groupe sanguin $A B O$ sont introduites sur un fichier local à l'aide d'un matériel PC compatible et d'un logiciel fournis par GMFT (Greffe de Moelle-France-Transplant) et transmis à l'ordinateur central VAX 8250 géré par l'Inserm U93 [7]. Les données sont transmises par télématique et interrogation directe à partir des PC compatibles au VAX 8250 durant la nuit avec transfert en retour des nouvelles données vers les PC.

\section{Résultats}

Les volontaires se répartissent en $44 \%$ d'hommes et $56 \%$ de femmes $18 \%$ d'entre eux ont moins de 26 ans et $63 \%$ moins de 36 ans. Le nombre de volontaires est très variable d'une région à l'autre sans rapport avec la densité de la population locale. Il dépend de la présence de structures mises en place localement et surtout de la volonté d'informer de cette possibilité de don par des familles de malades. Au mois de septembre 1987, 270 malades typés en $H L A-A, B, C$ et $D R$ constituaient le "fichier malades ". L'interrogation est faite toutes les trois semaines; $50 \%$ des malades appartiennent à un centre de l'Île-de-France et $50 \%$ à un centre de province. Environ $50 \%$ des malades sont atteints de leucémie myéloïde chronique.

Seulement 48 malades (17\%) n'ont pas de donneur HLA AB identique; 12 (4\%) ont de 26 à 46 donneurs HLA AB identiques. Si après une interrogation, il existe un donneur potentiel identique en $H L A$ $A$ et $B$, celui-ci est typé pour HLADR. L'identité HLA $A, B$ et DR ne suffit pas. Une enquête réalisée dans un laboratoire de la région parisienne montre que seuls $33 \%$ des malades ayant un ou plusieurs donneurs HLA AB et DR identiques ont une culture mixte lymphocytaire négative (identité HLAD). Parmi les malades ayant un donneur HLA AB DR et D identique, 8 ont déjà bénéficié d'une greffe de cellules médullaires: un cas d'aplasie, quatre cas de leucémie myéloïde chronique, deux cas de leucémie aiguë lymphoblastique de l'enfant et un cas de leucémie aiguë myéloblastique.

\section{Discussion}

A ce stade de développement du fichier des volontaires au don de moelle osseuse, il convient d'envisager d'une part de faire une information plus centrée sur les obligations d'un tel consentement puisque globalement plus de $20 \%$ des volontaires potentiels ne se présentent pas à la convocation pour typage HLA classe I. Ceci est peutêtre dû au fait que les délais sont encore trop longs entre la décision $d u$ volontaire et la convocation mais pour une bonne part la déci- sion pouvait être trop rapide et émotionnelle.

Il convient d'autre part d'uniformiser au plan européen les logiciels de gestion des fichiers afin qu'un échange puisse être envisagé rapidement. En effet une étude de B. Bradley [8] conclut qu'un fichier de 100000 volontaires permettrait de trouver dans $47 \%$ des cas un donneur HLA-AB, DR et D identique. Le projet s'oriente donc vers l'ouverture européenne.

C. Raffoux Greffe de moelle France-Transplant

\section{Références}

1. Dupont B, O'Reilly RJ, Pollack MS Good RA. I'se of HLA Genotypically different donors in bone marrow transplantation. Transplant Proc 1979; 11: 219-24.

2. O'Reilly RJ, Dupont B, Pahwa S. Reconstitution in severe combined immunodeficiency by transplantation of marrow from an unrelated donor. $N$ Engl J Med 1977; $297: 1311-8$

3. Hows JM, Yin JL, Marsh J, et al. Histocompatible unrelated volunteers donors compared with HLA non-identical family donors for marrow transplantation. Helsinki : XIth International Congress of the Transplantation Society, 1986 (abstr.). 4. Gale P. Potential utilization of a national HLA-Typed donor pool for bone marrow transplantation. Transplantation $1986 ; 42: 54-7$.

5. McCullough J, Rogers G, Dahl R, Therkelsen D, Kanstra L. Development and operation of a program to obtain volunteer bone marrow donors unrelated to the patient. Transfusion 1986; $26: 315$. 23.

6. McElligou MC, Menitove JE, Aster $\mathrm{H}$. Recruitment of unrelated persons as bone marrow donors. A prelimenary experience. Transfusion 1986; 26 : 309-14.

7. Raffoux C, Gluckman E., Busson M, Prevost P. Development of a national volunteer donor file for bone marrow transplantation. Chamonix: XIVth annual meeting of the EBMT IV meeting, EBMT nurses group, 1988

8. Bradley BA, Gilks MR, Gore SM, Klouda PR. How many HLA typed volunteer donors for bone marrow transplantation (BMT) are needed to provide an effective service? Bone Marrow Transplant $1987 ; 2$ (suppl. 1): 79. 